\title{
Simulation of the competition among traditional and on-demand software vendors
}

\author{
Luisanna Cocco, Giulio Concas and Michele Marchesi
}

\begin{abstract}
In this work, we propose a simulation model to study the software market, and in particular the competition among onpremise and on-demand vendors of customer relationship management software. In this market two kinds of vendors, on-premise and on-demand vendors, and corporate customers interact. Vendors have an initial capital and a specific number of developers. They invest a fraction of their capital in their product quality and try to sell their products making a profit. Corporate customers buy software products for their employees, trying to minimize their cost and maximize their utility. The proposed model is able to reproduce the real trends of the market as regards both the price of software and the market shares of the vendors, despite there is relatively little empirical evidence available about the various mechanisms at play, and few experimental data. Our model could be used as a tool to forecast future market trends, or to plan business policies of investment and pricing. Any firm could calibrate this model depending on its past business trends in order to obtain simple guidelines to follow in the planning of business winning strategies.
\end{abstract}

\section{Keywords}

Software as a service, perpetual licensing, pricing models, simulation model, heterogeneous agents, market strategy

\section{Introduction}

In recent years, the contractual relationship between software vendors and corporate customers has been deeply transformed due to diverse factors, both economic and technological. Software appears overpriced and the common thought of customers is of paying a too high price for software, which also supports low value processes or unused processes of a vendor. Customer willingness to pay is decreased and the opposition to the purchase of updates and of upgrades has increased. Nowadays, corporate customers know that they cannot become an efficient enterprize with the traditional software approach, and so pricing models based on periodic payments, in particular software as a service (SaaS), are strongly gaining ground.

SaaS, also called on-demand (OD) software, is a software application delivery model. Providers of SaaS have got the possession of the physical location, of the hardware, and of the system maintenance. Corporate users access software via the Internet from anywhere, and at any time. Users usually pay a subscription, and can run a single instance of a software on a robust infrastructure. SaaS applications are in fact delivered from a "multi-tenant" system; there is a single instance of software running, and many individuals or corporate customers use this system for their own necessities. According to a Gartner survey: ${ }^{1}$

\footnotetext{
"more than 95 percent of organizations expect to maintain or increase their investments in software as a service (SaaS), and more than one-third have migration projects under way from on-premises to SaaS."
}

These data are extracted from a survey made in June and July 2011 on 525 organizations in nine countries, spanning 12 vertical industries. The aim of this research was to understand the trends for SaaS adoption in enterprizes. Enterprize users declared to have adopted SaaS mainly for the easy and speed of deployment and for its cost-effectiveness, even if more than of one-third of respondents complain, for example, the limited integration with existing systems and the network instability.

Dipartimento di Ingegneria Elettrica ed Elettronica, University of Cagliari, Italy

\section{Corresponding author:}

Luisanna Cocco, Dipartimento di Ingegneria Elettrica ed Elettronica,

University of Cagliari, Piazza D'Armi, 09I23 Cagliari, Italy.

Email: luisanna.cocco@diee.unica.it 
This research also found that industries that are not using or considering SaaS solutions so far, are planning to use and consider SaaS solutions in a near future. In short, service oriented software is changing the world of software, and new software pricing trends are by now a reality that we cannot disregard.

Among the 10 leading publicly listed software vendors in US, Europe and Asia with respect to the presence in the OD software segment, we can cite Google, Intuit, Microsoft, NetSuite, Oracle, Salesforce.com for US west coast; RightNow for US mid-west; Sage and SAP for Europe; and finally CDC for Asia. ${ }^{2}$ Among OD software applications offered by these 10 top vendors, customer relationship management (CRM), enterprize resources planning (ERP), accounting, e-commerce, human resources management (HRM), and supply chain management (SCM) can be reported.

In this paper we propose a model to analyze the pricing trend, and the overall business sustainability of SaaS. The model is based on the heterogeneous agents approach, ${ }^{3}$ where the agents represent the software vendors of a vertical software market, and their corporate customers. The vendors follow either traditional licensed software pricing approach, or SaaS approach. The model is simulated to assess the behavior of price trends and of vendors' market shares. In particular, the model analyses a CRM software market in which vendors of perpetual licenses and vendors of SaaS compete. The model parameters are taken from other papers in the literature, or from real market data.

The paper is organized as follows. In Section 2 we discuss other works related to this paper, in Section 3 we present our model in detail. Section 4 presents the results of the simulations, including a sensitivity analysis and a Monte Carlo analysis in the Sections 4.2 and 4.3, respectively. The conclusions of the paper are reported in Section 5.

\section{Related work}

Many models appeared on the subject of simulation of the software market. They face the issue from very different points of view, and gave specific and precious insights into the development of this work. Among others, we quote the papers by Mustonen, ${ }^{4}$ Bonaccorsi and Rossi, ${ }^{5}$ Bitzer and Schröder, ${ }^{6}$ Lin, ${ }^{7}$ Economides and Katsamakas, ${ }^{8}$ and Cocco et al. ${ }^{9-11}$

Bonaccorsi and Rossi ${ }^{5}$ proposed a simulation model in order to identify the relevant factors in the diffusion of open source, modeling the adoption decision of heterogeneous interacting agents. Lin $^{7}$ studied how users' skill and network effects can influence the software market, characterized by proprietary firms, and open-source firms. Bitzer and Schröder ${ }^{6}$ analyzed the influence of entry and competition of open-source software on innovation and technological progress in software markets. Economides and
Katsamakas ${ }^{8}$ compared industry structures based on an open-source platform with those based on a proprietary platform, analyzing the competition and the industry implication in terms of pricing, sales, profitability, and social welfare. The work of Mustonen ${ }^{4}$ explained the simultaneous existence of commercial alternatives to copylefted programs and why commercial alternatives to copyleft programs may not exist. The works of Cocco et al. ${ }^{9-11}$ analyzed the influence of Free Libre Open Source Software (FLOSS) firms in the software market, nowadays mostly dominated by large proprietary firms.

The research papers that gave specific and precious insights to develop our model are the works of Haruvy et al. ${ }^{12}$ and Ghosh, ${ }^{13}$ that suggested some variables of our model, and the works of Bucholtz, ${ }^{14}$ Yankee Group, ${ }^{15}$ and SugarCRM $^{16}$ that suggested the orders of magnitude of the prices of the different software solutions.

Haruvy et al. ${ }^{12}$ examine a model in a monopoly setting where open source code is free but complements another product sold commercially. The work of Ghosh ${ }^{13}$ studied instead the role of FLOSS in the economy, its direct impact on the ICT sector, and its indirect impact on the ICTrelated sector. Bucholtz ${ }^{14}$ provided a comparative price analysis of four leading CRM solutions for midmarket organization. The CRM solutions included in this analysis are Microsoft Dynamics CRM 2011, Sage SalesLogix, Salesforce.com, and SugarCRM. Yankee Group ${ }^{15}$ provided a comparative analysis about total cost of ownership of a hosted versus premise-based CRM solution. In particular, they analyzed the costs of SalesLogix and salesforce.com implementations. Finally, in SugarCRM ${ }^{16}$ the pricing options for CRM products are analyzed.

\section{Model}

In this work, we propose a model to represent the time evolution of a vertical software market, i.e. a market where a specific kind of software applications compete. In particular, we refer to the CRM software market, which we derive empirical data from. We stipulate that relevant changes to the market can occur on a monthly basis, so our time steps are of 1 month. The main modeled agents are software vendors and customers.

There are two kinds of vendors, producing either onpremise (OP) or OD products, which we call OP and OD vendors, respectively. Vendors have an initial capital and number of developers (which for the sake of simplicity does not change with time), they decide how much to invest in their product quality, and try to sell their products making a profit. We model their business and their main characteristics. ${ }^{2,17,18}$

Customers represent corporate customers, which buy software products for their employees, trying to minimize their cost and maximize their quality. 


\section{I. $O P$ and $O D$ vendors}

All vendors produce a software application, characterized by quality $Q(t)$ and price $P(t)$. OP vendors represent traditional software vendors, whose product is run on hardware located in the premises of the customer. OP vendors also produce a secondary product, representing the services of assistance, consultancy and maintenance associated with the use of the primary product. The secondary product is complementary to the primary one, so the purchase of the primary cannot be separated from the acquisition of the secondary one.

The primary product of $i$ th OP vendor has a price equal to $P_{i}^{O P p}(t)$, paid by consumers at the moment of the purchase. The secondary product has a price equal to $P_{i}^{O P s}(t)$, paid by users monthly.

In the case of OD vendors, there is no distinction between primary and secondary product. The marketed product is only one. It is a complete product that includes delivery, support, and on-going maintenance. The pricing model adopted by these vendors entails periodic payments $P_{i}^{O D}(t)$ on a monthly subscription base (for $i$ th OD vendor). Corporate customers pay a monthly fee based on usage (the number of people at the company using the software).

The $i$ th vendor enters the market at time $t_{i}^{E}$. In our simulations, OP vendors enter at the start of the simulation, whereas OD vendors enter later.

\subsection{Investment policies}

Each $i$ th vendor enters the market with a fixed number of developers, $N_{i}$, and an amount of initial capital $A_{i}=k_{I N} N_{i}$. The initial capital available per developer, $k_{I N}$ is higher for OD vendors, because they have to setup hardware and network for providing their services. We set $k_{I N}=50,000$ for OP vendors, and $k_{I N}=80,000$ for OD vendors, in a nominal currency, whose value is set close to US\$.

All vendors allocate a percentage $\beta$ of their capital for the initial development of their products. This is determined by a random draw, being $\beta$ a normal variable with average and standard deviation equal to 0.9 and 0.03 , respectively.

For OP vendors, the quantity $C_{i}\left(t_{i}^{E}\right)$ allocated to develop the product is given by

$$
C_{i}\left(t_{i}^{E}\right)=\beta A_{i}
$$

In the case of OD vendors, they bear the same costs of OP vendors, but need to invest also in hardware and network, therefore $C_{i}\left(t_{i}^{E}\right)$ is given by

$$
C_{i}\left(t_{i}^{E}\right)+C_{H, i}\left(t_{i}^{E}\right)=\beta A_{i}
$$

where $C_{H, i}\left(t_{i}^{E}\right)$ is the initial investment needed to set up the server farm. Consequently, the sum devoted to develop the initial OD product is: $C_{i}\left(t_{i}^{E}\right)=\beta A_{i}-C_{H, i}\left(t_{i}^{E}\right)$, where $C_{H, i}\left(t_{i}^{E}\right)=\alpha A_{i}$.
After the initial development, vendors use the labor of their developers to perform maintenance, and to develop new versions of their products. This costs to them $s N_{i}$ units of money each month, being $s$ the average developer's wage. We set $s=3000$ units of money for all vendors. In addition to this "standard" work, vendors also bear further costs aimed to increase their human capital, and thus the final quality of their products (see next section for a definition of "quality").

We stipulate that the investments made by vendors in order to update their software product quality are always a fixed percentage of the initial investment $C_{i}\left(t_{i}^{E}\right)$. This is because the initial investment determined the initial positioning in the market segment of the product, and the vendors try to keep this positioning throughout the simulation.

OP vendors update their products at time intervals $\Delta_{O P}$ equal to 18 months, investing in quality an amount of capital, $C_{U, i}(t)$, proportional to $C_{i}\left(t_{i}^{E}\right)$

$$
C_{U, i}(t)=m_{i}(t) C_{i}\left(t_{i}^{E}\right)
$$

In addition, each month OP vendors invest smaller amounts of capital, $C_{Q, i}$, to maintain product quality to an acceptable level. These investments are smaller than $C_{U, i}(t)$ and are defined by

$$
C_{Q, i}(t)=r_{i}(t) C_{i}\left(t_{i}^{E}\right)
$$

In the case of OD vendors, small amounts of capital $C_{Q, i}(t)$ are invested each month for maintaining product quality to an acceptable level and for the maintenance of the product, and small amounts of capital $C_{H, i}$ are invested in hardware and network. These amounts are again proportional to $C_{i}\left(t_{i}^{E}\right)$ and $C_{H, i}\left(t_{i}^{E}\right)$, respectively, and are defined in

$$
\begin{gathered}
C_{Q, i}(t)=r_{i}(t) C_{i}\left(t_{i}^{E}\right) \\
C_{H, i}(t)=w_{i}(t) C_{H, i}\left(t_{i}^{E}\right)
\end{gathered}
$$

The parameters $m_{i}, r_{i}$ and $w_{i}$, in equations (3)-(6), are normal variables (see Table 1 for their definition). All investments made by each company are equally distributed among the developers of the company, and increase their productivity by $h_{i}$.

Of course, companies invest only when their financial balance, $B_{i}(t)$, allows it, that is if $B_{i}(t)>0$. During the simulation, the expenses and the revenues are recorded for each vendor at each time step. The following equation shows the balance, $B_{i}(t)$, of the $i$ th vendor at time $t$ :

$$
\begin{aligned}
& B_{i}(t)=A_{i}+\sum_{k=t_{i}^{E}}^{t} E_{i}(k) *\left(1-v_{i}\right)- \\
& \sum_{k=t_{i}^{E}}^{t}\left[C_{U, i}(k)+C_{Q, i}(k)+s N_{i}-C_{H, i}(k)-I_{i}(k)\right]
\end{aligned}
$$


Table I. Values of the parameters of OP and OD vendor equations.

\begin{tabular}{|c|c|c|c|}
\hline Parameter & Description & On-Premise Vendors & On-Demand Vendors \\
\hline$k_{I N}$ & Initial capital per developer: & 50,000 & 80,000 \\
\hline$N_{i}$ & Number of developers for each vendor: & $N(\mu=135, \sigma=21)$ & $N(|35| 1)$, \\
\hline$\beta$ & $\begin{array}{l}\text { Fraction of capital invested for initial development } \\
\text { of the product: }\end{array}$ & & $N(0.45,0.02)$ \\
\hline$\alpha_{i}$ & Fraction of capital invested for hardware expenses: & - & $N(0.45,0.02)$ \\
\hline$s$ & Developer's wage: & & 3000 \\
\hline$r_{i}$ & Fraction of capital invested monthly: & $N\left(2 \times 10^{-3}, 3.3 \times 10^{-4}\right)$ & $N\left(3 \times 10^{-3}, 3.3 \times 10^{-4}\right)$ \\
\hline$\omega_{i}$ & $\begin{array}{l}\text { Fraction of capital invested monthly in hardware } \\
\text { and network expenses: }\end{array}$ & - & $N\left(4.5 \times 10^{-4}, 2.5 \times 10^{-5}\right)$ \\
\hline$m_{i}(t)$ & Fraction of capital invested for updating the products: & $N\left(5 \times 10^{-3}, 3.3 \times 10^{-4}\right)$ & - \\
\hline
\end{tabular}

Note: $N(\mu, \sigma)$ represents a normal distribution with average $\mu$ and standard deviation $\sigma$.

where most of the symbols are defined in the previous part of this section; $E_{i}(t)$ indicates the earnings of the $i$ th vendor at time $t, v_{i}$ indicates the percentage of earnings allocated to the charter members as dividends, and $I_{i}(t)$ represents the bank loan received at time $t$. We set $v_{i}=0.5$ for all vendors.

When the balance in (7) is less than zero, the vendor requests a bank loan. If this loan exceeds its initial capital, the company goes bankrupt. This happens only to vendors active in the market for at least six months.

\subsection{Quality of the software products}

Each software product is characterized by a specific quality $Q_{i}$. The quality reflects all of the software features that contribute to its perceived value, for instance functionality, reliability, efficiency, and usability. The quality of the products is defined as in the work of Haruvy et al. ${ }^{12}$ by using the differential equation

$$
\frac{d Q_{i}}{d t}=h_{i}(t) N_{i}-\delta Q_{i}
$$

where $h_{i}(t)$ is the per-capita human capital of the $i$ th vendor at time $t$, and $\delta$ is the quality depreciation rate equal to 0.03 per month, as given by Haruvy et al. ${ }^{12}$

The per-capita human capital, $h_{i}(t)$, models the individual productivity and is defined in the following equation, as given by Ghosh: ${ }^{13}$

$$
\frac{d h_{i}}{d t}=\left[\pi\left(u h_{i}\right)^{\gamma}\left(\nu S_{Q, i}\right)^{1-\gamma}\right]
$$

where:

- $\pi$ is a constant parameter reflecting the productivity of human capital accumulation process;

- $u$ is the fraction of time spent on human capital formation;
- $\quad \gamma$ weighs the fraction of human capital and information and communications technology (ICT) capital in human capital accumulation process;

- $\quad \nu$ is an implicit measure of the use of ICT in human capital formation;

- $S_{Q, i}$ is the capital per employee invested in the quality of the product from $t_{i}^{E}$ to current time $t$.

The parameters $\pi, u, \gamma$ and $\nu$ are constant parameters equal to $0.025,0.1,0.9$ and 0.1 , respectively, as provided by Ghosh. ${ }^{13}$

\subsection{Pricing of the software products}

Software vendors adopt pricing strategies fundamentally different from those of other industries. ${ }^{19,20}$ Indeed, software products differ by traditional products because they do not lose quality as a result of their usage, although there is a loss of value over time. Moreover, a high production cost is needed to produce the first copy, but the cost of further copies is negligible.

Another feature of software products is the existence of network effects. The value of software depends on its properties, but also on the number of users who adopt it: the larger the number of users, the better it is for all of the users. The network effects have a significant impact on pricing strategies of software vendors, who use them to hinder the market entry to competitors, and bind the customers to the current provider, with high switching costs.

To model vendors' pricing strategies, we propose pricing mechanisms which vary over time for the two types of vendors. The initial price of the product, set at time $t_{i}^{E}$, when the vendors enter the market, is given by the following pricing rules. The initial price, $P_{i}^{O P p}\left(t_{i}^{E}\right)$, of primary OP product of $i$ th OP vendor is defined as

$$
P_{i}^{O P p}\left(t_{i}^{E}\right)=\left[1+\phi_{i}\right] \frac{N_{f}\left(t_{i}^{E}\right) C_{i}\left(t_{i}^{E}\right)}{K_{i} N_{C}\left(t_{i}^{E}\right)}
$$

where: 
- $\emptyset_{i}$ is the percentage of profit that $i$ th vendor wishes to obtain; it is characterized by a normal distribution with average equal to 0.15 and standard deviation equal to 0.02 ;

- $C_{i}$ is the investment made by the company to enter the market;

- $\quad N_{f}$ is the number of companies in the market;

- $N_{C}$ is the number of customers in the market;

- $K_{i}$ models the amortization period of the production costs of the software for $i$ th vendor.

This definition assumes an ideal market in which the customers choose the vendors with the same probability. Here $P_{i}^{O P p}(t)$ is a license fee per end-user and defines also the price of secondary product, $P_{i}^{O P S}$, calculated as a percentage of $P_{i}^{O P p}(t)$.

The price of the OD products is defined as follows:

$$
P_{i}^{O D}\left(t_{i}^{E}\right)=\left[1+\phi_{i}\right]\left(\frac{C_{i}\left(t_{i}^{E}\right)}{K_{i} N_{C}\left(t_{i}^{E}\right)}\right)
$$

This price is a fee per end-user/month, aimed to obtain a realistic price similar to the real ones.

Equations (10) and (11) stem from the literature on the pricing schemes for SaaS and perpetual software ${ }^{20,21,22}$ and define the price at the time a vendor enters the market as in Cocco et al. ${ }^{10,11}$ Over time, the vendors determine the price of their products following a pricing mechanism called derivative-follower pricing scheme, described by Rohitratana and Altmann. ${ }^{22}$ It assumes that vendors decide to increase, to decrease, or not to vary their price, depending only on their revenues, and neglecting the market conditions and the demand of customers.

In this pricing scheme, vendors evaluate their revenues at fixed time intervals $\Delta t$ (set to 3 months). If the revenue at $t+\Delta t$ is higher than the revenue at $t$ of at least $5 \%$, all is fine and the vendor does not vary its pricing mechanism. Otherwise, if this condition is not satisfied, vendors must decide whether to keep the price constant, to increase the price trying to increase the revenues, or to decrease it trying to attract new customers. This is decided with a random draw of equal probability. The increase or decrease of the price is a constant quantity, $\zeta$, that for OP vendors is $\zeta=30$, whereas for OD vendors is $\zeta=2$.

Once price has been changed, it is maintained for at least 6 months, before a different pricing strategy can be applied. All of the prices set can never be lower than a threshold $P_{T h}$, representing the minimum price required to amortize production and periodic costs. In other words, it is not possible to set a price that cannot at least amortize the investment and operational costs.

Parameters $K_{i}$ (in Equations (10) and (11)) are calibrated setting them equal to the years in which the vendors expect to have a positive return on the investments. The amortization period must be determined on the basis of an amortization plan, taking into account the useful life of the software to be adopted and its cost. The parameters $K_{i}$ referring to the price of the OD products assume higher values than those of OP products. This is because, in the case of OD vendors, revenues are collected throughout the course of the subscription and not up-front, and consequently having a long amortization period becomes very important.

In order to better reproduce the real pricing mechanisms, we included a discount mechanism for purchases of OP products over a given number of copies, as suggested by Bucholtz. ${ }^{14}$ In addition, we took into account the costs of switching from OP products to OD products and vice versa. According to the Yankee Group, ${ }^{15}$ we defined them as the sum of the cost of the OP or OD product and of the costs incurred for basic customization and integration and/ or for ongoing operational support.

Regarding the upgrades, their prices depend on how much customization and integration of the application must be made to the software application. It is not easy to compare OP with OD solutions because the upgrades in OD solutions occur on the average three or four times a year, and thus their cost can be accounted for together with the maintenance cost. For this reason, we assumed that these prices are included in the secondary price in the OP case, and in the monthly fee in the OD case.

Note that the prices described above are prices per enduser per month. In the OP case, the corporate users pay according to the number of their end-users. In the OD case, they pay according to a pay as you go model, that calculates the real usage of software, which can vary over time. Hence, the corporate customers pay for the number of endusers who actually use the software. This number varies in the range between $\left(e_{u}-0.3 e_{u}\right)$ and $e_{u}$ being equal to the number of the corporate customers's end-users.

Of course, the pricing mechanism just described is a simplification of the real pricing mechanisms. Its calibration aims to obtain price values similar to real prices. ${ }^{14,15,23,24}$

\subsection{The customers}

In our model, the customers are corporate customers. The number of customers in the market, $N_{C}$, is equal to 40,000 . Every customer buys software for an installation $e_{u}$ that may vary between 100 and 1000 end-users. In fact, we decided to study a software market aimed at small and medium corporate customers. ${ }^{14}$

The budget allocated by customers to buy software is not limited, so there is no constraint forcing to buy only low-cost software. The pricing remains, of course, an important factor influencing the choice, as explained below. Initially, a customer evaluates all products on the market, chooses a product using a utility function and buys it; in this way, it binds itself to a vendor.

From time to time, customers can compare their products to other products in the market, to verify if their 
current product is the most convenient choice. At each time $t$, a number of customers $N_{r-e}=\frac{N_{C}}{12}$ is drawn at random to re-evaluate their purchase choice. This means that this re-evaluation happens, on the average, each year. When new $i$ th vendor enters the market, at time $t=t_{i}^{E}$, a substantial number of users $N_{r-e}=\frac{N_{C}}{3}$ is drawn at random, representing the customers interested to evaluate the new product just launched.

The customers drawn to re-evaluate their and other products look for the best product in the market. They compare the values of the utility functions associated with their products to those of all the other products in the market, and in the end they decide whether to replace or not their products.

In general, a utility function $U$ denotes the subjective evaluation about the attitude of an asset or service to meet an economic need, i.e. a use value. ${ }^{5,7,25}$ In our model it depends on: quality, price, attitude toward the new SaaS pricing model $\eta$, network effects $\varepsilon$, switching costs $\rho$, and on a multiplicative normal noise $\chi$.

For the $j$ th customer and for the primary product of the $i$ th vendor, $U$ is defined as

$$
\begin{aligned}
U_{j, i}(t)= & a_{Q} Q_{i}^{O P p}(t, \chi)-a_{P} P_{i}^{O P p}(t, \chi)+\eta(t, \chi) \\
& +a_{N e} \varepsilon_{j, i}(t, \chi)-a_{S c} \rho_{j, i}(t, \chi)
\end{aligned}
$$

The first two terms take into account the quality and the price of the products. The third term, $\eta$, models the customer feeling toward a new SaaS technology, so it models how customers evaluate the benefits and the risks associated with the adoption of OD products, as illustrated in section 3. The value of $\eta$ varies with the size of corporate customers. ${ }^{2,26}$ These references affirm that the greatest potential growth in SaaS comes from the smaller business areas. In this area the companies typically invest in software less than large enterprizes, and are generally running outdated solutions. Consequently, they see OD solutions as a way of increasing their competitivity without facing the high up-front costs typical of OP solutions. For this reason, $\eta \in N(0.1,0.02)$ for customers with a number of end users, $e_{u}$, higher than 500, whereas $\eta \in N(0.2,0.02)$ for customers with a number of end users, $e_{u}$, lower than 500 , being $N(\mu, \sigma)$ a normal distribution with average $\mu$ and standard deviation $\sigma$.

The network effect term $\varepsilon$ depends on the number of customers of a software solution. It describes the fact that the utility that a consumer derives from the consumption of a product depends (positively or negatively) on the number of other customers who consume the same product. In our case, the larger the number of customers of the $i$ th vendor, the better it is for them. The network effect is defined as follows:

- if $N_{c, i}(t)<N_{c, M 1}$, the number of customers is too low and there is no network effect;
- if $N_{c, i}(t)>N_{c, M 2}$, the software usage is very spread and network effect saturates to 1 ;

- if $N_{c, M 1} \leqslant N_{c, i}(t) \leqslant N_{c, M 2}$, then

$$
\varepsilon=\frac{\left(N_{c, i}(t)-N_{c, M 1}\right)}{N_{C, M 2}-N_{c, M 1}}
$$

where:

- $\quad N_{c, i}(t)$ is the number of customers of $i$-th vendor at time $t>0$,

- $N_{c, M 1}=a N_{C}$ is the threshold above which the network effect starts; $a=0.2$, and

- $N_{c, M 2}=b N_{C}$ is the threshold above which the network effect saturates to $1 ; b=0.7$.

The random term $\chi$ represents a multiplicative normal noise, with average 0 and standard deviation 0.1 . It perturbs the generic term $f(x)$, in (12), in the following way:

- if $\chi>0$, then $f(x, \chi)=f(x)(1+\chi)$;

- if $\chi<0$, then $f(x, \chi)=\frac{f(x)}{1+|\chi|}$.

The last term in Equation (12) accounts for the switching cost $\rho$. Note that the values of quality, price and switching costs must be normalized. To this purpose, we proceed in the following way. Assuming that the minimum price is $P_{\min }$ and the maximum price is $P_{\max }$, the normalized price $P_{i}^{N}$ is a function of the generic price, $P_{i}$, and is given by the following equation:

$$
P_{i}^{N}=\frac{P_{i}-P_{\min }}{P_{\max }-P_{\min }}
$$

In this way, all of the quantities in the utility function are in the range $[0,1]$.

All of the terms in the utility function are multiplied by the $a_{x}$ coefficients, each computed for each customer through a random draw from a normal distribution $N(\mu, \sigma)$, with average $\mu=0$ and standard deviation $\sigma=0.1$.

In conclusion, the utility function values calculated by the $j$ th customer for each $i$ th vendor, are the sum of the features of the $i$ th product, weighed by the coefficients $a_{x}$ and by the random term $\chi$. In this way, each user has a different perception of the products in the market, with respect to the other customers.

\section{Simulation results}

The foregoing model was run to study the software market, in particular using empirical data from the CRM software market, by using a simulation-based approach. Given the limited quality of the available data, it makes sense to analyze the business trends in an ideal CRM market, where 
Table 2. Parameter values for the base run.

\begin{tabular}{lll}
\hline Parameter & Description & Base run \\
\hline$a_{x}$ & Coefficients of the utility function & $N\left(0,10^{-1}\right)$ \\
$\chi$ & Normal noise & $N\left(0,10^{-2}\right)$ \\
$\varepsilon$ & Network effect & $\left(N_{c, i}(t)-N_{c, M I}\right)$ \\
$N_{c, M 2}-N_{c, M I}$ & $N(0.1,0.02)$ & $e_{u}>500$ \\
$N(0.2,0.02)$ & $e_{u}<500$ \\
$\rho$ & Attitude toward OD products & $\left\{\begin{array}{l}N(0)-O P, \rho_{O P-O P}=0.28 * P_{i}^{O P p} \\
\rho_{O D} \\
\rho_{O P-O D}, \rho_{O D-O D}=0.1 * P_{i}^{O D}\end{array}\right.$
\end{tabular}

Note: $\rho_{x-y}$ indicates the switching costs from $x$ firm to $y$ firm.

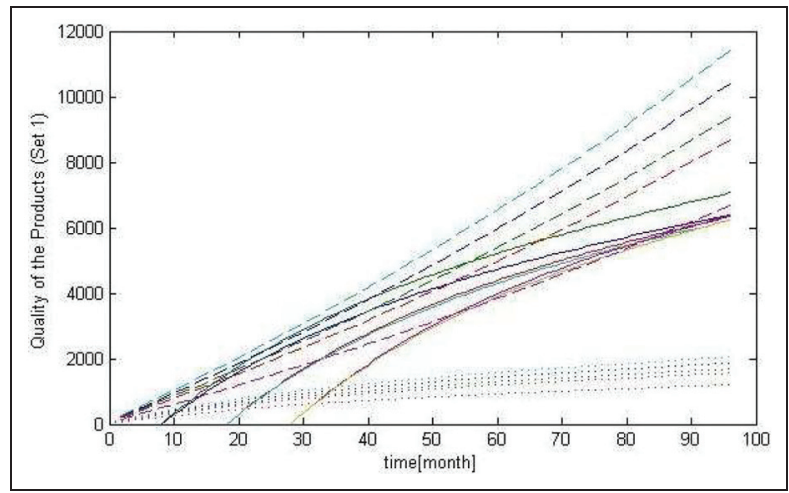

Figure I. Quality of OP primary (dashed lines), OP secondary (dotted lines) and SaaS (OD; solid lines) products (base run).

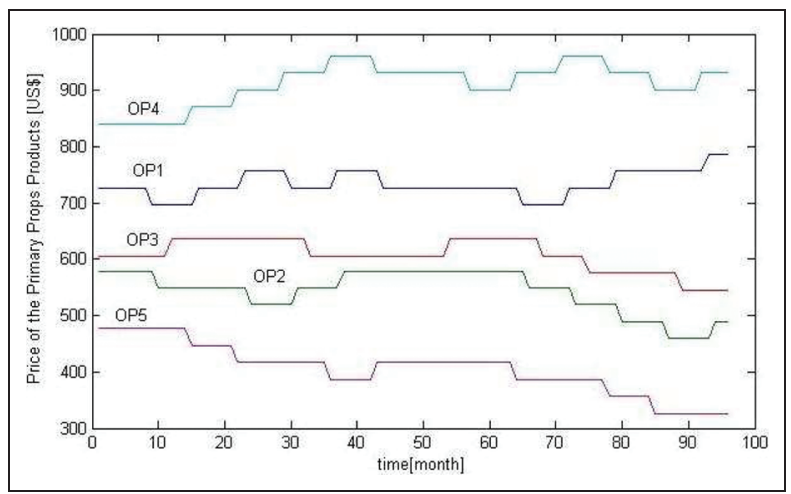

Figure 2. Prices of OP primary products (base run); the prices of secondary products are about a quarter of the prices shown.

the parameter values are chosen following empirical data and studies found in the literature. We call this setting the base run. The parameter values of this set are described in Tables 1 and 2. We studied the competition among OP and OD vendors, analyzing the behavior of the market over a simulation interval equal to $T=96$ time steps (months), hence considering a span of 8 years, enough to ascertain the main trends of a vertical software market.

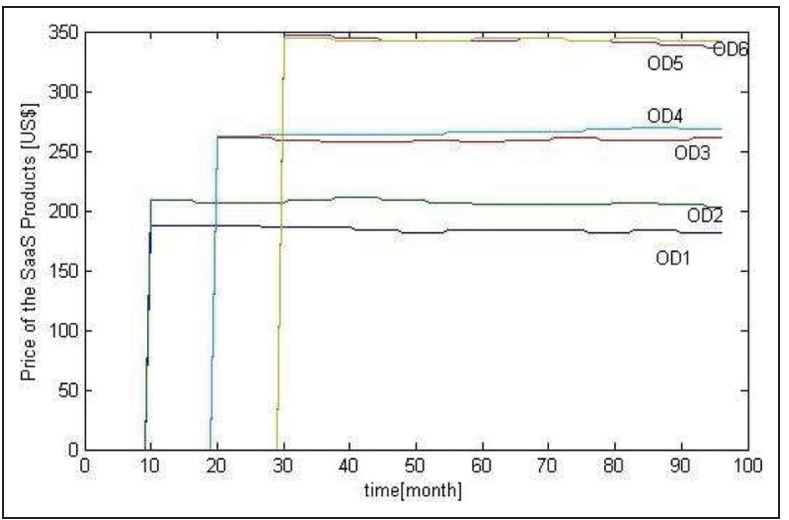

Figure 3. Price of SaaS (OD) products (base run).

\section{I. Base run results}

In the base run, we run the model setting all of its parameters as reported in the previous sections and in Table 2. The values reported are taken from the literature, from market analysis and some of them are set in order to obtain prices that match the real prices in the market. We analyzed the competition between five OP CRM vendors, which enter the market at $t_{\text {entry }}=0$, and six OD CRM vendors, which enter the market in pairs, at $t_{i}^{E}=10,20$, and 30 . This matches the real situation, where the entry of OD vendors occurred after that of OP ones. The results obtained in this simulation setting are reported in Figures $1-4$ and in Table 3.

The figures show the time trends of quality and prices, for the two kinds of CRM products (OP and OD), and the distribution of customers among vendors. Table 3 shows the number of customers at $t=t_{i}^{E}$ and at $t=T$, the end of simulation, for all of the different vendors in the market. These data highlight how OD vendors are able to conquer quite a large market share since the moment they enter the market, and to compete with OP vendors.

Figure 1 shows how the quality of OP and products varies over time. We can note that the values of OP quality is 


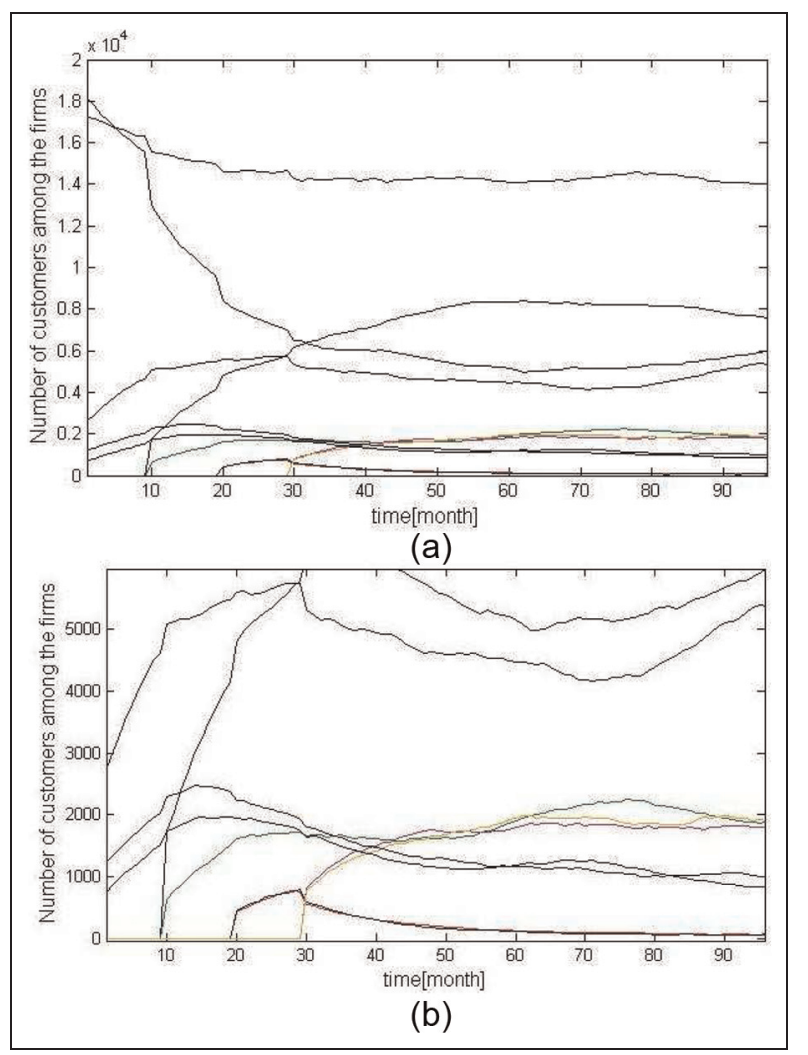

Figure 4. Customer distributions among OP (dotted lines) and SaaS (solid lines) vendors (base run). The Figure below (b) expands the $y$-axis to highlight the customer distributions with smaller values.

larger than that of OD products, in agreement with the fact that OP solutions are more customized than OD ones. ${ }^{27}$ The quality values are very high because of the chosen values of the initial investments and human capital. However, the reported considerations about quality do not change if we assume lower values of these parameters. The steady increase in "quality" for all vendors reflects the money continuously poured by vendors to improve the functionalities of their products, and the fact that, in this simulation, no vendor is driven out of the market, going bankrupt, although a couple of OD vendors end with a very low number of customers. Note that the investment policies used in this work stem from analysis of the market and from our experience. In fact, knowing the actual values of the investments made by the real firms is practically impossible. For this reason, most of our assumptions aim to calibrate the model in order to obtain realistic orders of magnitude of prices, a target that was achieved.

Figures 2 and 3 show the prices of OP and OD products, respectively. The prices of OP CRM vary between about 300 and 1000 for primary products, and between about 80 and 260 for secondary products (not shown in the figure because they are simply a fraction of the price of the corresponding primary product); the prices of OD CRM vary between about 180 and 350, matching the real prices (in US\$) in the market. OD prices look much stabler than OP ones, which anyway do not vary so much. Clearly, the model settings and the rule that prices cannot drop below the actual costs yield such a stable behavior. Other settings might yield more dynamic price trends.

In Figure 4, we show the time trends of the distributions of customers among the vendors. The initial (when the vendors enter the market) and final data of this graph have been already presented in Table 3. OP vendors get the whole market share in the beginning. Then, their customers decrease when OD vendors enter the market, because many of them decide to switch to OD software. All vendors are able to stay in the market for 96 time steps (months), although two OD vendors in the end are left with very a small market share, namely with 58 and 69 customers only (see Table 3).

Note that, due to the lack of experimental data, all parameters of this simulation have been calibrated in order to obtain prices that do not differ too much from those that nowadays we observe in the real CRM software market. The market trends we obtain reflect the real trends of the market, where only few vendors are able to survive with big market shares, whereas the remaining survive with much smaller market shares.

Table 3. Number of customers of all vendors in the base run, when the vendor enters the market and at the end of the simulation, $T$.

\begin{tabular}{lllll}
\hline Vendor & \multicolumn{2}{l}{ OP customers } & & OD customers \\
\cline { 2 - 4 } & at $t=0$ & at $t=T$ & at $t=t_{i}^{E}$ & 2055 \\
at $t=T$ \\
1 & 2657 & 5361 & 769 & 7578 \\
3 & 1219 & 1009 & 481 & 1890 \\
4 & 713 & 831 & 509 & 69 \\
5 & 17,259 & 14,018 & 931 & 1789 \\
6 & 18,152 & 5981 & 876 & 1913 \\
Customers & 40,000 & 27,200 & 5,621 & 13,297 \\
\hline
\end{tabular}


In particular, at $t=T$ one OP vendor gets a market share equal to $35 \%$ at $t=T$, and another OD vendor gets a market share equal to $19 \%$. Two other OP vendors get market shares equal to $13 \%$ and $15 \%$. All other seven vendors together get a market share of only $18 \%$. For the sake of brevity, we are not able to report the results of other simulations, made varying key parameters such as the leaning to invest in quality, or the price variations decided when the market share decreases. We report instead in the following section a sensitivity analysis of the model with respect to some of its parameters.

\subsection{Sensitivity analysis}

To study the sensitivity of the model to the various terms in the utility function, and hence its influence of the parameters, $\chi, \rho, \eta, \varepsilon$ and $a_{j}$ on the survival of the vendors, we run several simulation sets. In the following, we briefly describe the obtained results.

The analysis performed highlighted that the presence of $\chi$ in the base run has accomplished the purpose for which it was introduced, keeping all the vendors active in the market, without ousting many of them since the beginning. In fact, the multiplicative normal noise $\chi$ impacts on the distribution of customers among the vendors. Decreasing the value of $\chi$, the number of vendors with no customers or with a very low number of customers increases, even if the total market shares of OD and OP vendors do not vary. So, parameter $\chi$ influences the distribution of customers among the vendors, independently of their type.

As regards coefficients $a_{x}$, the analysis shows that their calibration in the base run does not privilege any type of vendor. However, the customers' purchase choices can be heavily influenced by the setting of these coefficients. For instance, if we define them as belonging to the normal distribution $N(0.1,0.1)$ (compared to $N(0,0.1)$ used in the base run), the purchase choices become directed towards the least expensive products, because most coefficients become positive. Therefore, OP products, which have a higher price than OD products, experience a reduction of their utility function values, with respect to their competitors. In contrast, if we define $a_{x}$ coefficients as belonging to the normal distribution $N(-0.1,0.1)$, the purchase choices are mainly directed towards the most expensive products, because most coefficients become negative.

The performed analysis shows also that the network effect coefficient, $\varepsilon$, the switching costs $\rho$, and the parameter $\eta$ which models the customer feeling toward new technologies, do not significantly influence the distribution of customers. For instance, as regards parameter $\eta$, the purchase choice for OD products persists also decreasing the value of this parameter, which by definition increases the value of the utility function of OD products.

A further analysis was performed to investigate the sensitivity of the model to the value of the simulation period
$T$, the number of vendors $N_{f}$, the number of customers $N_{C}$, and the number of customers $N_{r-e}$ drawn at every time for re-evaluating their purchase choices. The analysis performed highlighted that these parameters have a low influence on the distribution of customers among the vendors, and hence on the total market shares got by the two types of vendors.

\subsection{Monte Carlo analysis}

In addition to the sensitivity analysis, a Monte Carlo (mc) analysis was performed to the purpose of confirming the results of the sensitivity analysis, and assessing the robustness of our model.

For computational reasons, and given that no significant difference in the outputs of the model has been found with respect to the total number of customers and vendors, and with respect to the number of customers drawn to reevaluate their purchase choices, the mc analysis was performed running a smaller version of the base run, called Run1.

In this set, we assumed a total number of customer equal to 20,000 , a number of OP vendors equal to 3 , a number of OD vendors equal to 4 , and finally a number of customers drawn to re-evaluate their purchase choices equal to $N_{r-c}=N_{C} / 24$, with all of these numbers being smaller than those of the base run.

In Tables 4 and 5, we describe all simulation sets run, defining the parameter values which vary from set to set. Runl was run in order to assess the robustness of the proposed model and the results presented in Section 4.1. Instead Run1.1-Run1.7 were run with the aim of confirming the results obtained from the sensitivity analysis.

We repeated each simulation set 100 times with the same initial conditions, but with different seeds of the random number generator. For each mc run and for each vendor we recorded the customer number at the entry time and at the exit time. In addition, for each mc run we computed the number of surviving vendors. In Tables 6 and 7 , we show the results of the mc analysis. In particular, we report the 25 th, 50 th, 75 th, 85 th and 95 th percentiles of the number of customers for the survivor firms and the average number of survivor vendors across all mc simulations.

The mc analysis confirmed both the robustness of the model and the results of the sensitivity analysis. As regards the robustness of the model, overall all performed simulations presented a consistent behavior, confirming the robustness and the validity of the results presented in Section 4.1. In fact, the parameter settings of the Runl (see Table 4) do not privilege heavily any type of vendor and only a few vendors are able to survive with big market shares, whereas the remaining survive with much smaller market shares (see Table 6).

Concerning the sensitivity analysis, we studied the impact of the coefficients $a_{x}$ and of the parameters $\varepsilon, \eta$ 
Table 4. Parameter values for Run I, Run I.I, Run I.2, RunI.3, and Run I.4.

\begin{tabular}{|c|c|c|c|c|c|}
\hline Parameter & Run I & Run I.I & Run 1.2 & Run 1.3 & Run 1.4 \\
\hline$a_{x}$ & $N\left(0,10^{-1}\right)$ & $N\left(10^{-1}, 10^{-1}\right)$ & $N\left(-10^{-1}, 10^{-1}\right)$ & $*$ & $*$ \\
\hline$\chi$ & $N\left(0,10^{-2}\right)$ & $*$ & * & $N\left(0,10^{-4}\right)$ & 0 \\
\hline$\varepsilon$ & $\left(N_{c, i}(t)-N_{c, M I}\right)$ & $*$ & $*$ & $*$ & * \\
\hline$\eta$ & $\begin{array}{l}N_{C, M 2}-N_{c, M I} \\
\begin{cases}N(0.1,0.02) & e_{u}>500 \\
N(0.2,0.02) & e_{u}<500\end{cases} \end{array}$ & $*$ & $*$ & $*$ & $*$ \\
\hline$\rho$ & $\left\{\begin{array}{c}\rho_{O D-O P}, \rho_{O P-O P}=0.28 * P_{i}^{O P p} \\
\rho_{O P-O D}, \rho_{O D-O D}=0.1 * P_{i}^{O D}\end{array}\right.$ & $*$ & $*$ & $*$ & $*$ \\
\hline
\end{tabular}

Note: * indicates that the parameter values are defined as in Run I.

Here $\rho_{x-y}$ indicates the switching costs from $x$ firm to $y$ firm.

Table 5. Parameter values for Runl, Run I.5, Run I.6, and RunI.7.

\begin{tabular}{|c|c|c|c|c|}
\hline Parameter & Run I & Run I.5 & Run I.6 & RunI.7 \\
\hline$a_{x}$ & $N\left(0,10^{-1}\right)$ & $*$ & $*$ & $*$ \\
\hline$\hat{\chi}$ & $N\left(0,10^{-2}\right)$ & $*$ & * & $*$ \\
\hline$\varepsilon$ & $\frac{\left(N_{c, i}(t)-N_{c, M 1}\right)}{N_{c, M 2}-N_{c, M 1}}$ & 0 & $*$ & $*$ \\
\hline$\eta$ & $\begin{cases}N(0.1,0.02) & e_{u}>500 \\
N(0.2,0.02) & e_{u}<500\end{cases}$ & $*$ & 0 & $*$ \\
\hline$\rho$ & $\begin{array}{l}\rho_{O D-O P}, \rho_{O P-O P}=0.28 * P_{i}^{O P p} \\
\rho_{O P-O D}, \rho_{O D-O D}=0.1 * P_{i}^{O D}\end{array}$ & $*$ & * & 0 \\
\hline
\end{tabular}

Note: * indicates that the parameter values are defined as in Runl.

$\rho_{x-y}$ indicates the switching costs from $x$ firm to $y$ firm.

Table 6. Monte Carlo analysis: percentiles of the number of customers and total average number of survivor vendors for Run I, RunI.I, Run I.2, Run I.3, and RunI.4.

\begin{tabular}{|c|c|c|c|c|c|c|c|c|c|c|c|}
\hline \multirow[t]{2}{*}{ Firm } & \multirow[t]{2}{*}{ Percentile } & \multicolumn{2}{|l|}{ Run I } & \multicolumn{2}{|l|}{ RunI.I } & \multicolumn{2}{|l|}{ Run I.2 } & \multicolumn{2}{|l|}{ Run I.3 } & \multicolumn{2}{|l|}{ Run I.4 } \\
\hline & & $t=t^{E}$ & $t=T$ & $t=t^{E}$ & $t=T$ & $t=t^{E}$ & $t=T$ & $t=t^{E}$ & $t=T$ & $t=t^{E}$ & $t=T$ \\
\hline \multirow[t]{5}{*}{ OP } & $P_{0.25}$ & 1445 & 2132 & 667 & 639 & 402 & 1644 & 0 & 106 & 0 & 169 \\
\hline & $P_{0.50}$ & 5491 & 3767 & 4648 & 1403 & 6142 & 4362 & 3869 & 3437 & 2465 & 3407 \\
\hline & $P_{0.75}$ & 10,692 & 4870 & II,240 & 1795 & 11,223 & 10,317 & 13,243 & 5921 & 14,907 & 5151 \\
\hline & $P_{0.85}$ & $\mid 4,772$ & 5329 & 15,473 & 2214 & 13,366 & 12,068 & 16,362 & 7527 & 18,036 & 6766 \\
\hline & $P_{0.95}$ & 16,206 & 7785 & 16,822 & 2615 & 18,547 & 13,920 & 18,242 & 9538 & 18,998 & 7940 \\
\hline \multirow[t]{5}{*}{ OD } & $P_{0.25}$ & 1 & 756 & 75 & 1776 & I & 209 & 0 & 227 & 0 & 138 \\
\hline & $P_{0.50}$ & 196 & 2262 & 740 & 3966 & 2 & 612 & 2 & 2298 & 2 & 1290 \\
\hline & $P_{0.75}$ & 1839 & 3698 & 2515 & 6111 & 4 & 1150 & 1427 & 4319 & 573 & 5084 \\
\hline & $P_{0.85}$ & 4576 & 4949 & 3733 & 7058 & 1347 & $|76|$ & 3345 & 4959 & 3608 & 6396 \\
\hline & $P_{0.95}$ & 5828 & 5893 & 5316 & 9159 & 4393 & 2403 & 5420 & 5965 & 6110 & 8022 \\
\hline \multicolumn{2}{|c|}{ Survivor average } & - & 7 & - & 7 & - & 6.9 & - & 6.1 & - & 5.8 \\
\hline
\end{tabular}

and $\rho$ on the customers' purchase choices. We found that, the customers' purchase choices can be heavily influenced by the setting of coefficients $a_{x}$ and of the parameter $\chi$, but by the setting of parameters $\varepsilon, \eta$, and $\rho$.

Indeed, a rise of the coefficients $a_{x}$ from $N\left(0,10^{-1}\right)$ to $N\left(10^{-1}, 10^{-1}\right)$ (see Run1 and Run1.1 in Table 4) directs the purchase choices towards the least-expensive products, and consequently the OD firms conquest much higher market shares than OP firms (see Run1.1 in Table 6). In contrast, a drop of the coefficients $a_{x}$ from $N\left(0,10^{-1}\right)$ to $N\left(-10^{-1}, 10^{-1}\right)$ (see Runl and Run1.2 in Table 4) directs the purchase choices towards the most expensive products, and as a result the OP firms conquest much higher market shares than OD firms (see Run1.2 in Table 6).

The parameter $\chi$ was introduced in the utility function with the aim of keeping all of the firms active in the market. An its drop from $N\left(0,10^{-2}\right)$ to $N\left(0,10^{-4}\right)$ (see Runl and Run1.3 in Table 4) or from $N\left(0,10^{-2}\right)$ to 0 (see Run1 
Table 7. Monte Carlo analysis: percentiles of the number of customers and total average number of survivor vendors for Run I, RunI.5, RunI.6, and RunI.7.

\begin{tabular}{|c|c|c|c|c|c|c|c|c|c|}
\hline \multirow[t]{2}{*}{ Firm } & \multirow[t]{2}{*}{ Percentile } & \multicolumn{2}{|l|}{ Run I } & \multicolumn{2}{|l|}{ Run I.5 } & \multicolumn{2}{|l|}{ Run I.6 } & \multicolumn{2}{|l|}{ Run I.7 } \\
\hline & & $t=t^{E}$ & $t=T$ & $\overline{t=t^{E}}$ & $\overline{t=T}$ & $\overline{t=t^{E}}$ & $t=T$ & $\overline{t=t^{E}}$ & $\overline{t=T}$ \\
\hline \multirow[t]{5}{*}{ OP } & $P_{0.25}$ & 1445 & 2132 & 1803 & 1497 & 769 & 1979 & 2433 & 1323 \\
\hline & $P_{0.50}$ & 5491 & 3767 & 4638 & 3509 & 3153 & 3545 & 6195 & 2914 \\
\hline & $P_{0.75}$ & 10,692 & 4870 & 13,237 & 5474 & 10,657 & 5127 & 10,279 & 4826 \\
\hline & $P_{0.85}$ & 14,772 & 5329 & 14,447 & 6214 & 16,483 & 6027 & 11,765 & 6680 \\
\hline & $P_{0.95}$ & 16,206 & 7785 & 16,374 & 7815 & 18,977 & 7579 & 17,282 & 8309 \\
\hline \multirow[t]{5}{*}{ OD } & $P_{0.25}$ & 1 & 756 & 2 & 1004 & 0 & 878 & 3 & 858 \\
\hline & $P_{0.50}$ & 196 & 2262 & 683 & 2235 & 0 & 2647 & 681 & 2437 \\
\hline & $P_{0.75}$ & 1839 & 3698 & 3080 & 3921 & 880 & 4053 & 2360 & 3609 \\
\hline & $P_{0.85}$ & 4576 & 4949 & 3606 & 4139 & 2137 & 4679 & 3976 & 4557 \\
\hline & $P_{0.95}$ & 5828 & 5893 & 4878 & 4906 & 5798 & 5324 & 4867 & 5613 \\
\hline \multicolumn{2}{|c|}{ Survivor Average } & - & 7 & - & 7 & - & 6.9 & - & 7 \\
\hline
\end{tabular}

and Run1.4 in Table 4) implies the exit from the market of some vendors, and hence an average number of survivor vendors smaller than seven (see Run1.3 and Run1.4 in Table 6).

Finally, as regards the parameters $\varepsilon, \eta$ and $\rho$, their setting in Run 1 does not imply any significant impact on the customers' purchase choices. Indeed, also a drop of these parameters to 0 (see Run1.5 and Runl.7 in Table 5) does not imply any significant impact on the customers' purchase choices, and the results of these runs do not show any substantial difference with respect to those of the Runl.

To conclude, we highlight that the model is fairly complex and is based on several parameters, most of them essential to a realistic software market model. However, even if the results obviously depend on the chosen parameters' values, the performed analysis demonstrated that the chosen parameters' values for the base run can be considered broadly correct, because they do not force the purchase choices of customers toward a specific vendor or toward a specific type of vendor.

\section{Conclusions and future work}

In this paper we have presented a simulation-based approach to analyze one of the latest trends in the software market: SaaS. To the best of the authors' knowledge, this is the first time that the software market has been modeled detailing the investment and pricing policies and the purchase preferences of customers.

Since there is relatively little empirical evidence available about the strengths of the various mechanisms on hand, and few experimental data to initialize or validate the simulations, many of the model's parameters were calibrated according to existing literature and market analysis. The proposed model was able to reproduce the actual trends of the market.
To study the sensitivity of the model to the different parameters and to justify the chosen parameter values, a sensitivity and a mc analysis were conducted. The sensitivity analysis confirmed how the outputs of the proposed model are more sensitive to the variations of some key parameters with respect to others. The mc analysis confirmed that the response of the model to different initial values of the parameters is stable, allowing us to generalize the considerations done for the base run presented.

In conclusion, we obtained a sufficiently realistic reproduction of a market, that follows the trends of the real market, concerning both the survival of the firms and the features of the products. Clearly, the presented model and test simulations are only a first step toward the direction of producing a robust and reliable model of the real market. They show the potentiality of our model, highlighting as OD software gains ground and attracts an increasing interest of the enterprizes' customers. Of course, more significant results could be obtained calibrating the model with more real data about pricing and investment policies of the vendors.

Our final goal is to develop a useful predictive model, able to forecast market trends, and to optimize investment and pricing policies. By using it, a vendor could use the past business trends of itself and of its competitors, in order to obtain optimal policy settings to be followed for a business winning strategy. Presently, we are working on getting more real data to calibrate the model, and on enhancements of it.

\section{Funding}

This research is supported by Regione Autonoma della Sardegna (RAS; Regional Law No. 7-2007, project CRP-17938 "LEAN $2.0 ")$. 


\section{References}

1. Pettey C and Van der Meulen R. Survey Reveals 95 Percent of Respondents Expect to Maintain or Grow Use of SaaS. Stamford: Gartner, October 2011.

2. Slowinski S, Nguyen R and Panicker S. Global software on demand. Shifting the balance of power. Societe Generale Cross Asset Research, September 2007.

3. Delli Gatti D, Gaffeo E, Gallegati M, et al. Emergent Macroeconomics: An Agent-Based Approach to Business Fluctuations. Berlin: Springer, 2008.

4. Mustonen M. Copyleft - the economics of Linux and other open source software. Informat Econ Policy 2003; 15(1): 99-121.

5. Bonaccorsi A and Rossi C. Why open source software can succeed. Res Policy 2003; 32: 1243-1258.

6. Bitzer J and Schröder PJH. The impact of entry and competition by open source software on innovation activity. EconWPA, December 2005, available at: http://EconPapers .repec.org/RePEc:wpa:wuwpio:0512001.

7. Lin L. Impact of user skills and network effects on the competition between open source and proprietary software. Electronic Commerce Res Appl 2008; 7(1): 68-81.

8. Economides $\mathrm{N}$ and Katsamakas E. Two-sided competition of proprietary vs. open source technology platforms and the implications for the software industry. Management Sci 2006; 52(7): 1057-1071.

9. Cocco L, Mannaro K, Concas G, et al. Study of the competition between proprietary software firms and free/libre open source software using a simulation model. In: Software Business (Lecture Notes in Business Information Processing, vol. 80). New York: Springer, 2011, pp. 56-69.

10. Cocco L, Concas G and Marchesi M. Agent-based modelling and simulation of the software market, including open source vendors. Int J Modell Sim 2014; 34(3): 113-119.

11. Cocco L, Concas G, Marchesi M and Destefanis G. Agentbased modelling and simulation of the software market, including open source vendors. $J$ Informat Technol Management 2013; 24(1): 13-25.

12. Haruvy E, Sethi SP and Zhou J. Open source development with a commercial complementary product or service. Product Operat Management 2008; 17(1): 29-43.

13. Ghosh RA. Study on the Economic Impact of Open Source Software on Innovation and the Competitiveness of the Information and Communication Technologies (ICT) Sector in the EU. UNU-MERIT, The Netherlands, 2006.

14. Bucholtz C. CRM Total Cost of Ownership: Fees, Subscriptions and Hidden Costs. CRM Outsiders and SugarSRM, USA, 2011.

15. Yankee Group. Understanding Total Cost of Ownership of a Hosted vs. Premises-Based CRM Solution. The Yankee Group, USA, 2004.

16. SugarCRM. CRM Total Cost of Ownership Comparing Open Source Solutions to Proprietary Solutions. SugarCRM, USA, 2005.

17. Dubey A and Wagle D. Delivering software as a service. The McKinsey Quarterly, Web exclusive, May 2007.

18. Thanawala M, Smith G, Dart J, et al. Oracle SaaS Platform: Building On-Demand Applications. Oracle, USA, September 2008.
19. Lehmann S and Buxmann P. Pricing strategies of software vendors. Business Inform Syst Eng 2009; 1(6): 452-462.

20. Dasgupta P and Das R. Dynamic pricing with limited competitor information in a multi-agent economy. In: 7th international conference on cooperative information systems, 2000, pp. 299-310.

21. Harmon R, Demirkan H, Hefley B and Auseklis N. Pricing strategies for information technology services: a value-based approach. In: Proceedings of the 42nd Hawaii international conference on system sciences, vol. 1, 2009, pp. 1-10.

22. Rohitratana J and Altmann J. Impact of pricing schemes on a market for software-as-a-service and perpetual software. Future Gen Comput Syst 2012; 28(8): 1328-1339.

23. CloudOne. Components of Total Cost of Ownership. CloudOne Corporation, Naperville, IL, USA, July 2010.

24. Software-as-a-Service Executive Council. Software-as-aService; A Comprehensive Look at the Total Cost of Ownership of Software Applications. Software and Information Industry Association, USA, 2006.

25. Berry ST. Estimating discrete-choice models of product differentiation. RAND J Econ 1994; 25(2): 242-262.

26. Etro F. The economic impact of cloud computing on business creation, employment and output in Europe. An application of the endogenous market structures approach to a GPT innovation. Rev Business Econ 2009; 54(2): 179-208.

27. OSF Global Services. SaaS CRM vs. On-Premise CRM: Which is the Rightchoice for Your Business?OSF Global Services, Canada, 2012.

\section{Author biographies}

Luisanna Cocco received the $\mathrm{PhD}$ degree in Electronic and Computer Engineering in 2013 and currently is PostDoc at Electric and Electronic Engineering Department of University of Cagliari. Her research interests concern modeling of economic and financial systems with heterogeneous agents (using complex system approach and agile methodologies), open-source software and engineering software, with particular attention to the market economy, the crypto-currency market, the software market and the software development.

Giulio Concas received the Laurea degree in Computer Science from the University of Pisa in 1989. From 1991 to 1999 he was graduate technician, in charge of the Scientific Calculus Area of IT Services Center of University of Cagliari. From 1995 he has performed several advanced consultancy activities in the field of computer science. He was a member of Local Linux User Group and an open-source supporter. From 1999 he was assistant professor of theory and applications of the Internet at the University of Cagliari. He has published more than 50 research papers in international journals and conferences. His research interests included Internet technology, service-oriented architectures, open-source software, agile methodologies, and software engineering. He suddenly passed away on 15 October 2014. 
Michele Marchesi received the Laurea degrees in electronic engineering and in mathematics from the University of Genoa in 1975 and 1980, respectively. He is professor of software engineering at the University of Cagliari. His research interests include software modeling using complex system approach, agile methodologies, open-source development and applications, modeling and simulation of financial markets and economic systems using heterogeneous interacting agents. He has published more than 200 papers in international journals, books and conferences. He has been the leader of several research projects, and is a consultant for various companies and public bodies. $\mathrm{He}$ is a member of the IEEE.

\section{Appendix}

\begin{tabular}{ll}
\hline Symbol & Description \\
\hline OP & On-Premise. \\
SaaS & Software as a Service. \\
OD & On-Demand. \\
CRM & Customer Relationship Management. \\
$P_{i}^{O P p}$ & Primary product price of the ith vendor. \\
$P_{i}^{O P S}$ & Secondary product price of the ith vendor. \\
$P_{i}^{O D}$ & OD product price of the ith vendor. \\
$Q_{i}$ & Product quality of the ith vendor. \\
$h_{i}$ & Per capita human capital of the ith vendor. \\
$\delta$ & Quality depreciation rate. \\
$t_{i}^{E}$ & Entry time of the ith vendor. \\
$N_{i}$ & Number of developers of the ith vendor. \\
$A_{i}$ & Initial capital of the ith vendor. \\
$k_{I N}$ & Initial capital per developer. \\
$C_{i}\left(t_{i}^{E}\right)$ & Initial investment of the ith vendor to \\
$C_{H, i}\left(t_{i}^{E}\right)$ & develop the product. \\
$C_{U, i}$ & Initial investment to set up the server \\
$C_{Q, i}$ & farm of the ith vendor. \\
$C_{H, i}$ & Investment of the ith vendor to update \\
$B_{i}$ & its product. \\
$E_{i}$ & Investment of the ith vendor to maintain \\
$l_{i}$ & product quality to an acceptable level. \\
$v_{i}$ & Investment of the ith vendor in hardware \\
$\mathrm{e}_{u}$ & and network. \\
$N_{r-e}$ & Financial balance of the ith vendor. \\
& Earnings of the ith vendor. \\
Bank loan of the ith vendor. \\
Percentage of the earnings of the ith vendor \\
allocated as dividends. \\
Number of end-users of corporate customers.
\end{tabular}

